\title{
Is Entamoeba Gingivalis a Risk Factor for Periodontal Diseases? A Case-
}

\section{Control Study}

\section{Sandy Shabaan Hassan, ${ }^{A}$ Gihane Gharib Madkour, ${ }^{B}$ Ramy Wahba Henin, C Selvia Wahib Fayek Gad, D Amany Ahmed Abd El-AalE,F}

A Lecturer, Department of Oral Medicine and Periodontology, Faculty of Dentistry, Fayoum University, Fayoum, Egypt.

${ }^{B}$ Associate Professor, Department of Oral Medicine and Periodontology, Faculty of Dentistry, Cairo University, Cairo, Egypt.

C Lecturer, Department of Medical Parasitology, Faculty of Medicine, Fayoum University, Fayoum, Egypt.

D Assistant Lecturer, Department of Medical Parasitology, Faculty of Medicine, Fayoum University, Fayoum, Egypt.

E Professor, Department of Medical Parasitology, Faculty of Medicine, Cairo University, Cairo, Egypt.

Accepted for publication: April 27, 2019

F Professor, Department of Medical Parasitology, Armed Forces

College of Medicine, Cairo, Egypt.

\section{Abstract}

Background: Entamoeba gingivalis was the first commensal parasite detected in the oral cavity of humans, and a high incidence has been reported in patients with poor oral hygiene. The current study aimed to investigate the association of Entamoeba gingivalis with gingivitis and periodontitis among Egyptian subjects. Methods: A total of 120 plaque samples were collected for this case-control study and were divided as follows: 40 plaque samples from gingivitis patients (group 1), 40 from stage II grade A and B periodontitis patients (group 2), and 40 samples from healthy volunteers (group 3). Diagnosis of parasitic stages relied on direct microscopic detection using permanent stains, trichrome stain, and hematoxylin and eosin (H\&E) stain, in addition to ocular micrometry to confirm the diagnosis. Results: The occurrence of Entamoeba gingivalis within the gingivitis group was significantly higher $(40 \%)$ than that observed in the control group (22.5\%), whereas the occurrence within the periodontitis group was $15 \%$. Samples from diseased subjects, regardless of immune status, were found to be moderately to severely affected with numerous parasitic nests, in contrast to a moderate near mild occurrence that was recorded in the healthy control group. Moreover, Entamoeba gingivalis occurrence was significantly higher (77.4\%) in subjects with bad oral hygiene. Conclusion: The results of the present study suggest a potential role for the neglected oral parasitic Entamoeba gingivalis, especially the intensively multiplying forms, in the pathogenesis of periodontal diseases. This certainly needs further elucidation on a larger scale to explore the basis behind such multiplication, which may be related to genetic variation or may be pathophysiological in origin.

Keywords: Entamoeba gingivalis; gingivitis; periodontitis; dental plaque biofilm; permanent stains.

\section{Introduction}

Gingivitis and periodontitis are the two mostly frequent plaque-induced inflammatory periodontal diseases influencing the periodontium, yet the etiology is not strongly evident. It is reported to be caused by microbial biofilms which form soft sticky dental plaques on the teeth. These biofilms release different immunogenic substances such as lipopolysaccharides with other virulence factors which initiate an immuno-inflammatory response. Consequently, inflammatory mediators including cytokines, chemokines, arachidonic acid 
metabolites, and proteolytic enzymes, jointly participate in tissue and bone destruction. Currently, there is enough evidence to suggest an association of a group of local microorganisms including Entamoeba gingivalis in such an oral pathological condition. ${ }^{1,2}$ Accordingly, this data opens a scientific gate for more research to explore the pathophysiology of gingivitis and periodontitis, adopting another point of view.

Entamoeba gingivalis is a unique Entamoeba species that often infects gingival tissues. It is documented to be more common in individuals with bad oral hygiene, as food debris and bacteria serve as nutrition for this parasite. Moreover, Entamoeba gingivalis has been detected in periodontal disease and in conditions of immune suppression. It particularly flourishes during suppurative inflammatory reactions owing to their favor for anaerobic settings. As Entamoeba gingivalis is similarly present in the oral cavity of healthy subjects, several authors consider this commensal to be opportunistic. Thus, it can proliferate in a gingival setting altered by periodontal disease. ${ }^{1,3}$

Studies reported that Entamoeba gingivalis contributes to the initiation and progression of gingivitis and periodontitis. These oral inflammatory conditions in turn, facilitate the proliferation of Entamoeba gingivalis. This endless loop may explain the occurrence of Entamoeba gingivalis in the saliva and dental plaque of gingivitis and periodontitis patients. 4,5 Therefore, the aim of the present study was to investigate the occurrence of Entamoeba gingivalis and its association with gingivitis and periodontitis among Egyptian subjects.

\section{Materials and Methods}

The present study was registered at ClinicalTrials.gov (Identifier: NCT03805724). The study was explained to the involved subjects and signed written consents approved by the research ethics committee were obtained.

\section{Study Population}

One hundred twenty subjects in total were enrolled in this case-control study (50 females and 70 males; age range: $35-55$ years; mean age of $40 \pm 5.25$ ). The subjects were divided into three groups: 40 patients who presented with gingivitis (group 1), 40 patients who presented with periodontitis (group 2), and 40 healthy control volunteers (group 3). A detailed medical history for each subject was obtained in accordance with the modified Cornell Medical Index. ${ }^{6}$ Written consent was obtained from each subject in accordance with the institutional guidelines after clarifying the study.

\section{Exclusion Criteria}

Individuals who received periodontal therapy in the six months prior to recruitment, pregnant females, and patients who had taken antibiotics or any other medication in the three months prior to recruitment were excluded from the present study.

\section{Clinical Examination}

Gingivitis and periodontitis patients were selected from the Outpatient's Clinic of the Department of Oral Medicine, Periodontology, and Diagnosis at the Faculty of Dentistry, Fayoum University. A clinical examination for all patients was performed and included the following periodontal parameters: plaque index $(\mathrm{PI})$, gingival index $(\mathrm{GI})$, probing depth $(\mathrm{PD})$, and clinical attachment level (CAL). These measurements were recorded by a single expert examiner at six sites for all teeth (mesiobuccal, midbuccal, distobuccal, distolingual, midlingual, and mesiolingual). The plaque index was assessed by measuring the presence or absence of a supragingival biofilm with a sweeping movement of the probe around the surfaces of all teeth. ${ }^{7}$ Marginal gingival bleeding was assessed via the GI. ${ }^{8}$ Probing depth was measured from the free-gingival margin to the base of the periodontal pocket, and CAL was measured from the cemento-enamel junction to the base of the periodontal pocket. Measurements were rounded to the nearest whole millimeter using the Michigan 0 probe with Williams' markings.

\section{Categorization of Subjects}

Subjects were categorized according to their clinical examination. Dental plaque biofilminduced gingivitis patients (group 1) had generalized gingivitis with an intact periodontium and no CAL, no radiographic bone loss, and bleeding on probing in more than $30 \%$ of teeth according to Murakami et al. 9 Stage II grade A and B generalized periodontitis patients (group 2) had PDs $\geq 3 \mathrm{~mm}$ and a CAL of 3-4 $\mathrm{mm}$ with more than $30 \%$ of teeth affected, according to the 2017 World Workshop on the Classification of Periodontal and Peri-Implant Diseases and Conditions. ${ }^{10}$ The control group (group 3) was selected from healthy subjects 
who attended the restorative dental clinic and had a clinically healthy gingiva with a nearly zero PI and $\mathrm{Gl}$, and $\mathrm{a} C A L$ and $P D<3 \mathrm{~mm}$.

\section{Sample Collection}

Plaque samples were collected after carefully drying and isolating the selected sites with cotton rolls. Supragingival plaque samples were collected using a periodontal probe in gingivitis patients and healthy controls. For periodontitis patients, sterile curettes were used to collect plaque samples from the selected periodontal pockets. Samples were immediately immersed in sterile Eppendorf tubes containing polyvinyl alcohol (PVA).

\section{Parasitological Examination}

The sample was diluted with PVA at room temperature $\left(25-28^{\circ} \mathrm{C}\right)$ and was stained with trichrome according to El-Dardiry et al. and hematoxylin and eosin (H\&E) following the procedures of Kim et al. 11,12 At least three smears were stained for proper parasitological examination, using $\times 40$ and oil immersion $\times 100$ magnifications. Entamoeba gingivalis parasites were identified by their shape based on the expansion of the pseudopodia and the presence of vacuoles, inclusions, and its characteristic nucleus. ${ }^{4}$ The parasitic stages were measured in accordance with Bailey et al. ${ }^{13}$ Objects seen under the microscope were measured using an eyepiece (ocular) micrometer that was calibrated against a stage micrometer in combination with a specific objective lens. The intensity of Entamoeba gingivalis occurrence was calculated according to Maybodi et al. with some modification. ${ }^{14}$ The parasitic stages were counted during microscopic examination and the severity of occurrence was calculated according to the following criteria: the presence of very few parasites was considered a mild occurrence ( 1 to 4 parasites), moderate occurrence was recorded when the number of parasitic stages was from 5-10, and severe occurrence was reported when Entamoeba gingivalis trophozoites were detected in nests or when more than 10 parasites were detected. Parasitic stages were counted in different smears, and the mean number was calculated to determine the severity of colonization. ${ }^{14}$

\section{Statistical Analysis}

Statistical analysis was performed using the IBM Statistical Package for the Social Sciences ${ }^{\circledR}$ (SPSS) (IBM Corp, Armonk, NY, USA, Release 16 for Microsoft Windows). Results were presented as frequencies, and the percent for the qualitative Chi-square test was used for comparing qualitative variables between groups. Fisher's exact test was used instead of the Chi-square test with two by two tables when expected cell count was less than five. The odds ratio (OR) with $95 \%$ confidence intervals was computed. A probability value $\leq 0.05$ was considered statistically significant.

\section{Sample Size Calculation}

Using the G*Power (Version 3.1.9.2) software, sample size was calculated at a power of $80 \%$ using $5 \%$ alpha $(\alpha)$ level and $20 \%$ beta $(\beta)$ level. A total of 120 subjects were required to be divided into three equal groups of 40 .

\section{$\underline{\text { Results }}$}

Demographic data and clinical periodontal parameters of all participating subjects in the 3 studied groups are shown in Table 1. Our results revealed that Entamoeba gingivalis was detected in 31 samples out of a total of 120 collected samples (Table 2). The positive cases in the studied subjects were as follows: $16(40 \%)$ of them were from the gingivitis group, 6 (15\%) from the periodontitis group, and the remaining $9(22.5 \%)$ subjects were from the control group.

The present work relied, not only on the characteristic morphological criteria to report positive findings concerning the Entamoeba gingivalis parasitic stage, but also, on the measurement of the detected stages using micrometry to confirm such findings. The detected trophozoites were observed with a single nucleus containing a small prominent central karyosome and a peripheral rim of chromatin, and finely granular cytoplasm. The size of the detected trophozoites in this study ranged from 10 to 16 $\mu \mathrm{m}$ (Figure 1). According to the categorization of Maybodi et al. ${ }^{14}$ which describes the intensity of occurrence, samples related to the diseased subjects irrespective of their immune status (diabetic or not) were found to be moderately to severely affected, while mild to moderate occurrence was recorded in the control group. Parasitic nests were observed in 17 out of the 22 positive cases from the diseased groups (Figure 2, C), while samples from the 9 positive cases in the control group did not demonstrate any nests.

Table 3 compares different stains used in the present work to detect Entamoeba gingivalis. With the H\&E stain, the cytoplasm of 
Table 1. Demographic data and clinical periodontal parameters for all subjects

\begin{tabular}{|c|c|c|c|}
\hline & Gingivitis ( $N=40)$ & Periodontitis $(\mathrm{N}=40)$ & Control $(\mathrm{N}=40)$ \\
\hline \multicolumn{4}{|l|}{ Age } \\
\hline$\leq 40$ years & $32(80 \%)$ & $10(25 \%)$ & $21(52.5 \%)$ \\
\hline$>40$ years & $8(20 \%)$ & $30(75 \%)$ & $19(47.5 \%)$ \\
\hline \multicolumn{4}{|l|}{ Sex } \\
\hline Female & $28(70 \%)$ & $16(40 \%)$ & $17(42.5 \%)$ \\
\hline Male & $12(30 \%)$ & $24(60 \%)$ & $23(57.5 \%)$ \\
\hline \multicolumn{4}{|l|}{ Residence } \\
\hline Urban & $23(57.5 \%)$ & $12(30 \%)$ & $19(47.5 \%)$ \\
\hline Rural & $17(42.5 \%)$ & $28(70 \%)$ & $21(52.5 \%)$ \\
\hline \multicolumn{4}{|l|}{ Occupation } \\
\hline High Paying Profession & $4(10 \%)$ & $3(7.5 \%)$ & $6(15 \%)$ \\
\hline Employee & $10(25 \%)$ & $5(12.5 \%)$ & $13(32.5 \%)$ \\
\hline Skilled Worker & $4(10 \%)$ & $11(27.5 \%)$ & $7(17.5 \%)$ \\
\hline Unemployed & $22(55 \%)$ & $21(52.5 \%)$ & $14(35 \%)$ \\
\hline \multicolumn{4}{|l|}{ Clinical Parameters } \\
\hline$\overline{\mathrm{PI}}$ & $1.59 \pm 0.42$ & $1.78 \pm 0.43$ & $0.51 \pm 0.11$ \\
\hline$\overline{\mathrm{GI}}$ & $1.64 \pm 0.39$ & $1.95 \pm 0.41$ & $0.14 \pm 0.09$ \\
\hline PD (mm) & $2.11 \pm 0.46$ & $4.13 \pm 0.57$ & $1.19 \pm 0.38$ \\
\hline $\mathrm{CAL}(\mathrm{mm})$ & 0 & $3.84 \pm 0.52$ & 0 \\
\hline
\end{tabular}

Table 2. Demographic data for Entamoeba gingivalis positive cases

\begin{tabular}{|c|c|c|c|}
\hline & $\begin{array}{c}\text { No. }(\%) \\
(\text { Total No.=120) }\end{array}$ & $\begin{array}{l}\text { No. }(\%) \text { Positive } \\
\text { (Total No.=31) }\end{array}$ & P Value \\
\hline \multicolumn{4}{|l|}{ Age } \\
\hline$\leq 40$ years & $61(50.8 \%)$ & $17(54.8 \%)$ & \multirow{2}{*}{0.76} \\
\hline$>40$ years & $59(49.2 \%)$ & $14(45.2 \%)$ & \\
\hline \multicolumn{4}{|l|}{ Sex } \\
\hline Female & $50(41.7 \%)$ & $21(67.7 \%)$ & \multirow{2}{*}{$0.001 *$} \\
\hline Male & $70(58.3 \%)$ & $10(32.3 \%)$ & \\
\hline \multicolumn{4}{|l|}{ Residence } \\
\hline Urban & $63(52.5 \%)$ & $14(45.2 \%)$ & \multirow{2}{*}{0.458} \\
\hline Rural & $57(47.5 \%)$ & $17(54.8 \%)$ & \\
\hline \multicolumn{4}{|l|}{ Level of education } \\
\hline High & $29(24.2 \%)$ & $7(22.6 \%)$ & \multirow{3}{*}{0.171} \\
\hline Middle & $42(35 \%)$ & $11(35.5 \%)$ & \\
\hline Low & $49(40.8 \%)$ & $13(41.9 \%)$ & \\
\hline \multicolumn{4}{|l|}{ Occupation } \\
\hline High Paying Profession & $19(15.8 \%)$ & $7(22.6 \%)$ & \multirow{3}{*}{0.083} \\
\hline Employee/Skilled Worker & $38(31.7 \%)$ & $7(22.6 \%)$ & \\
\hline Unemployed & $63(52.5 \%)$ & $17(54.8 \%)$ & \\
\hline
\end{tabular}

*Significant $(p \leq 0.05)$

the detected trophozoites appeared light pink in color, and the nucleus appeared dark red or violet in color. Cytoplasmic inclusions appeared more or less the same color as the nucleus, providing optimal contrast (Figure 1).
Pseudopodia were also observed in many of the identified trophozoites. With the trichrome stain, the cytoplasm of Entamoeba gingivalis appeared blue-green tinged with purple. The nuclei and inclusions were purple-red in most 
of the observed trophozoites (Figure 2, A \& B). Leuko-phagocytosis, which denotes the presence of engulfed white blood cells (WBCs), was observed in many Entamoeba gingivalis trophozoites as seen in Figure 2, C.

Figure 1. Entamoeba gingivalis trophozoites stained with H\&E stain
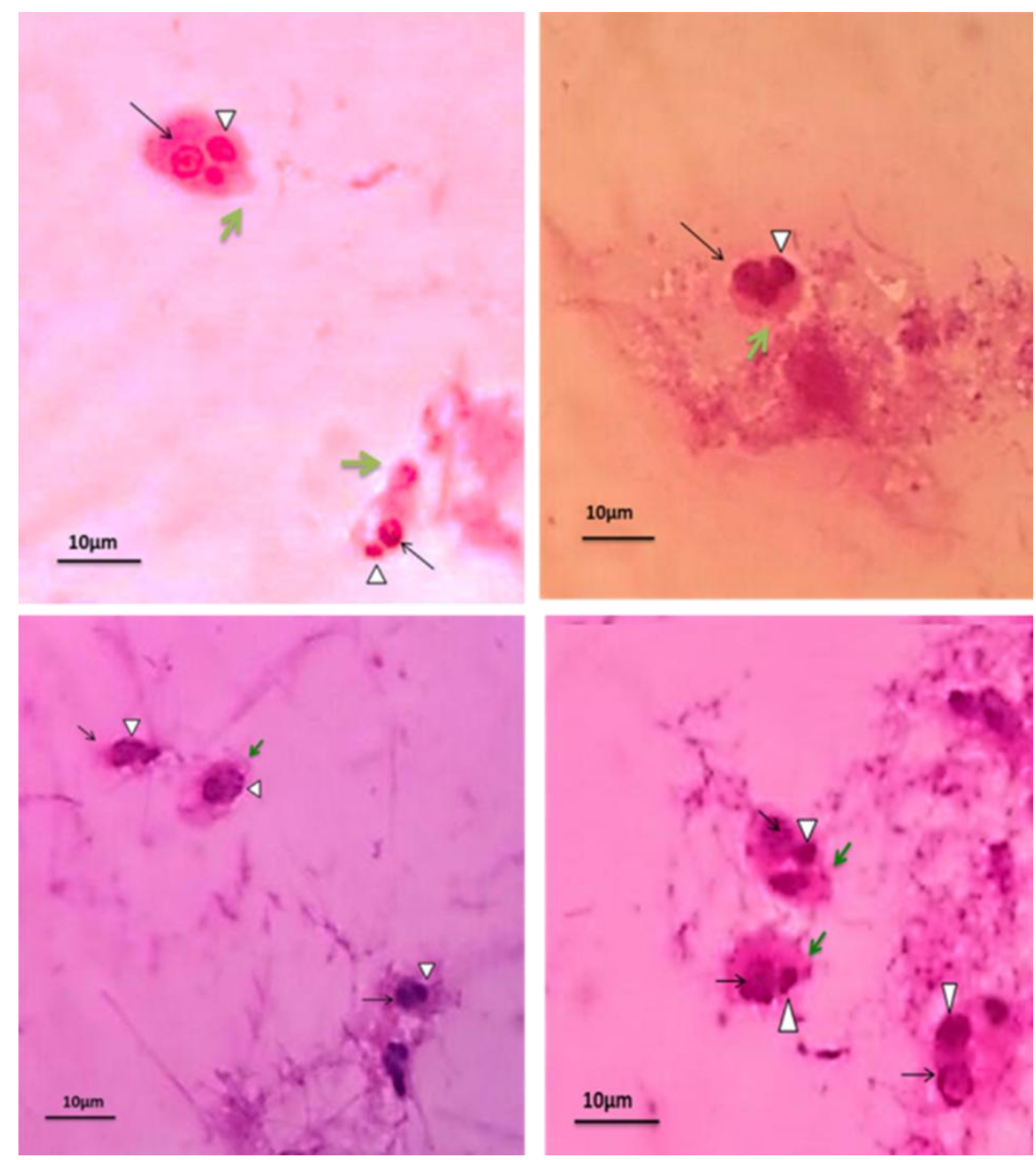

Note the characteristic nucleus with central karyosome (black arrows), cytoplasmic inclusions (arrow heads), and the pseudopodia (green arrows).

Figure 2. Entamoeba gingivalis trophozoites
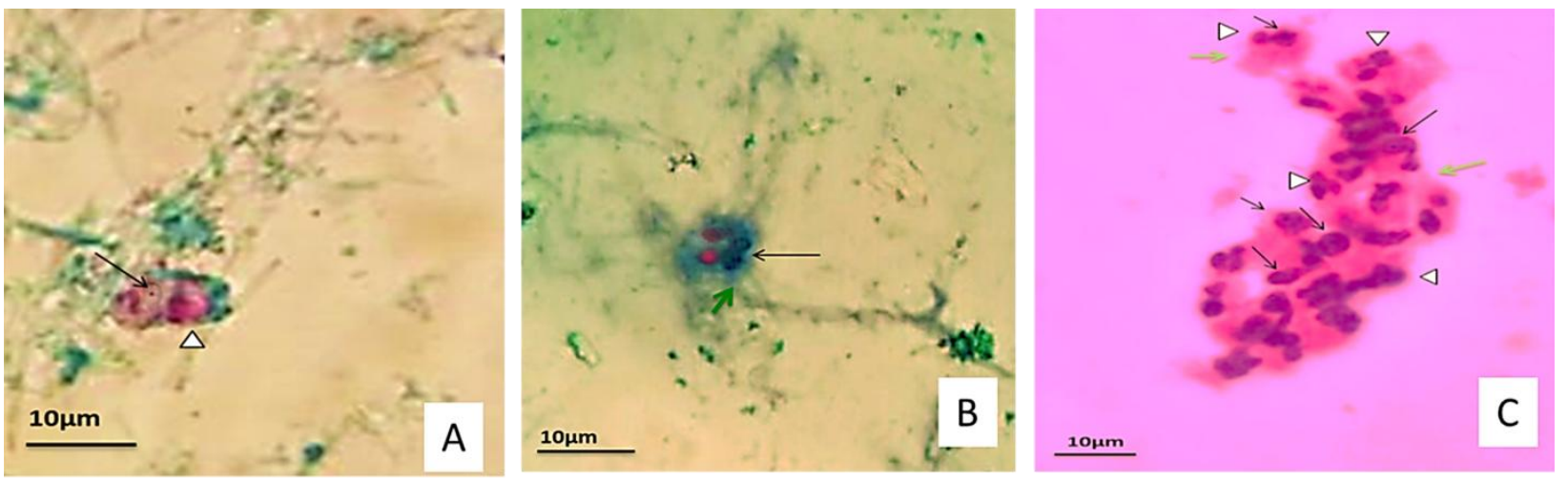

A\&B. Entamoeba gingivalis trophozoites stained with trichrome stain showing characteristic nucleus (arrows). The cytoplasm appears with mixed pink and green colors with reddish cytoplasmic inclusions (arrow head). Notice the scattered fungal infection. C. Nest of $E$. gingivalis trophozoites stained with $\mathrm{H} \& \mathrm{E}$ showing the characteristic nuclei (black arrows). Dark (violet) cytoplasmic inclusions (arrow heads) represent phagocytized WBCs. Pseudopodia are seen in some trophozoites (green arrows).

Table 4 shows the distribution of risk factors among all studied groups. Table 5 shows the distribution of risk factors among positive and negative cases. The effect of oral hygiene was statistically significant as the odds ratio was 1.57. The effect of smoking was statistically insignificant as the odds ratio was 0.54 . Regarding the effect of diabetes on positive 
cases, these values were statistically insignificant where the odds ratio was 0.55 . However, our study did not intend to target diabetes or any other cause of immune inadequacy for investigation.

Table 3. Comparison between different stains used to detect Entamoeba gingivalis

\begin{tabular}{|c|c|c|c|c|}
\hline Method of Detection & Gingivitis (N=40) & Periodontitis (N=40) & Controls (N=40) & P Value \\
\hline Trichrome stain & & & & \\
\hline $\mathrm{N}$ & 4 & 3 & 3 & 0.89 \\
\hline$\%$ & $10 \%$ & $7.5 \%$ & $7.5 \%$ & \\
\hline H\&E stain & & & 9 & $0.011^{*}$ \\
\hline $\mathrm{N}$ & 14 & 3 & $22.5 \%$ & \\
\hline$\%$ & $35 \%$ & $7.5 \%$ & 9 & $0.001^{*}$ \\
\hline Total & 16 & 6 & $22.5 \%$ & 0 \\
\hline $\mathrm{N}$ & $40 \%$ & $15 \%$ & & \\
\hline$\%$ & & & \\
\hline
\end{tabular}

*Significant $(p<0.05)$

Table 4. Risk factors among all studied groups

\begin{tabular}{|c|c|c|c|c|}
\hline & Gingivitis ( $N=40)$ & Periodontitis ( $N=40)$ & Control $(\mathrm{N}=40)$ & P Value \\
\hline \multicolumn{5}{|c|}{ Smoking } \\
\hline Yes & $30(75 \%)$ & $22(55 \%)$ & $30(75 \%)$ & \multirow{2}{*}{0.09} \\
\hline No & $10(25 \%)$ & $18(45 \%)$ & $10(25 \%)$ & \\
\hline \multicolumn{5}{|c|}{ Diabetes } \\
\hline Yes & $15(37.5 \%)$ & $15(37.5 \%)$ & $13(32.5 \%)$ & \multirow{2}{*}{0.865} \\
\hline No & $25(62.5 \%)$ & $25(62.5 \%)$ & $27(67.5 \%)$ & \\
\hline \multicolumn{5}{|c|}{ Oral Hygiene } \\
\hline Bad & $30(75 \%)$ & $38(95 \%)$ & $17(42.5 \%)$ & \multirow{2}{*}{$0.001 *$} \\
\hline Good & $10(25 \%)$ & $2(5 \%)$ & $23(57.5 \%)$ & \\
\hline
\end{tabular}

*Significant $(\mathrm{p} \leq 0.05)$

Table 5. Risk factors among the positive and negative cases

\begin{tabular}{|c|c|c|c|}
\hline & $\begin{array}{c}\text { No. }(\%) \text { Positive } \\
(\mathbf{N}=31)\end{array}$ & $\begin{array}{c}\text { No. }(\%) \text { Negative } \\
(\mathbf{N = 8 9 )}\end{array}$ & $\begin{array}{c}\text { Odds Ratio }(95 \% \\
\text { Confidence Interval) }\end{array}$ \\
\hline Smoking & $18(58.1 \%)$ & $64(71.9 \%)$ & $0.54(0.23-1.27)$ \\
\hline Yes & $13(41.9 \%)$ & $25(28.1 \%)$ & \\
\hline No & $6(16.1 \%)$ & $27(30.3 \%)$ & $0.55(0.2-1.5)$ \\
\hline Diabetes & $25(83.9 \%)$ & $62(69.7 \%)$ & \\
\hline Yes & $24(77.4 \%)$ & $61(68.5 \%)$ & $1.57(0.61-4.08)^{*}$ \\
\hline No & $7(22.6 \%)$ & $28(31.5 \%)$ & \\
\hline Oral Hygiene & & & \\
\hline Bad & & & \\
\hline Good & & & \\
\hline
\end{tabular}

*Statistically significant

\section{Discussion}

Periodontitis is a common oral disease affecting the global population yet its etiology is not fully determined. Researchers are still investigating the role of many factors such as microorganisms and environmental or genetic factors in the pathogenesis of this multifactorial disease. ${ }^{15}$

Periodontal lesions contain numerous neutrophils, bacteria, spirillae, spinning rods, and protozoa. The available information on periodontitis mainly focuses on the nature of its 
bacterial etiology. Parasites were not sought in many studies in spite of their potential role which cannot be ruled out. Entamoeba gingivalis, the first commensal found in the human oral cavity, might be a reason for the progression of periodontal diseases. Thus, we aimed to investigate the potential link between colonization of gingival crevices by this amoeba and gingivitis and/or periodontitis. ${ }^{16-18}$

Variable parasitological methods, strengthened by micrometry, were applied in the current study to confirm the diagnosis of Entamoeba gingivalis among our cases. Thirty one out of the 120 total subjects were evidenced to be positive for Entamoeba gingivalis occurence $(25.8 \%)$. Twenty six cases were diagnosed via H\&E stain which was significantly higher than those diagnosed using the trichrome stain (positive in only 10 cases). Therefore, H\&E stain identified $83.87 \%$ of the cases associated with Entamoeba gingivalis. Five samples did not obtain positive findings with $H \& E$, but the trophozoites were observed in samples stained with trichrome. This denotes the importance of examining more than one sample and more than one stain. In their study, Gardner et al. compared unstained wet mount with variable stains in order to identify Entamoeba species. ${ }^{19}$ They only recorded $4.8 \%$ positive results by unstained mount, while $58.5 \%$ were positive by permanent stains, indicating the importance of using permanent stains to confirm this parasitic occurrence in the oral cavity. The authors concluded that the direct unstained wet mount may be helpful in detecting cyst stages which was not a choice in our study as Entamoeba gingivalis does not undergo encystation. Additionally, the authors warned laboratorians about relying solely on the direct wet mount for identification of protozoan trophozoites. Instead, they recommended the use of permanent staining techniques which were reported to be much more effective for detecting and identifying protozoan trophozoites in different specimens. ${ }^{19}$

Goldsmid and Gericks reported that the occurrence rate for Entamoeba gingivalis diagnosed by contrast microscopy was $62.5 \%$ and $81.25 \%$ in permanent smears stained with iron hematoxylin. ${ }^{20}$ This is much higher than that reported in our study; the difference might be related to variation in study design, demographic variability, period of study, or the performed diagnostic techniques. Although AlNajar and Adnan conducted their study in Baghdad and reported a $28 \%$ colonization rate for Entamoeba gingivalis among their cases, their results are more or less similar to that reported by our study $\left(25.8 \%, 31\right.$ out of 120 cases). ${ }^{21}$

Regarding whether Entamoeba gingivalis is an infection or a commensal in the oral cavity, Glebski et al. conducted a research on students and disclosed the presence of this amoeba among $20 \%$ of healthy subjects. ${ }^{22}$ Their results are in accordance with our results as Entamoeba gingivalis was found in $22.5 \%$ of the healthy control group. In addition, Dao et al. found Entamoeba gingivalis in a larger number of cases (32\% of the healthy control group). ${ }^{23}$ However, Trim et al. recently used polymerase chain reaction (PCR) in a study that demonstrated a high incidence of Entamoeba gingivalis in individuals suffering from periodontitis yet it was not detected in any of the healthy gingival sites. ${ }^{24}$ This result was confirmed by Albuquerque et al. and Bonner et al. who reported that Entamoeba gingivalis is infrequently detected in healthy controls. ${ }^{17,18}$ This has led to a speculation that it might also be a contributing factor in the pathogenesis of periodontal diseases.

Results of the current study revealed that the occurrence of Entamoeba gingivalis among patients suffering from gingivitis, regardless of their immunological status was $40 \%$, and only $15 \%$ within the periodontitis group, yet with a higher intensity of infection. Moderate to almost mild infection was reported in the healthy control group. The rate observed in the periodontitis group is in accordance with that reported by several studies. 17,18,25,26 It has been suggested that these protozoa could affect the initiation, development, and progression of periodontal diseases.

It was not a matter of mere existence of parasitic stages in both healthy and diseased individuals. The results of our study showed a heightened intensity of colonization in the diseased groups compared to the control group which could possibly be related to a certain subtype of this parasite as suggested by Garcia et al. rather than the immune status of the individuals. ${ }^{5}$ In the present study, intensive colonization with numerous parasitic nests was demonstrated in immunocompetent diabetic cases. Thus, intensive proliferation was confirmed in the diseased cases, regardless of diabetic condition, indicating that this parasite may not be an opportunistic parasite.

Al-Saeed suggested that if Entamoeba gingivalis helps contribute to the development 
and progression of gingivitis and periodontitis, these diseases increasingly facilitate the proliferation of these protozoa. ${ }^{27}$ This interfering circle might explain the increased incidence of this amoeba in the dental plaque and saliva samples of patients with gingivitis and chronic periodontitis. The previous hypothesis is in accordance with our study and explains the presence of such a proliferating type of parasitic infection in the diseased groups in contrast to slowly multiplying forms in the healthy control group.

Putting into consideration some risk factors for periodontal diseases, we compared the patients' oral hygiene in the current research. There was a significant association between the occurrence of Entamoeba gingivalis and the level of hygiene as $77.4 \%$ of cases were of bad oral hygiene. This result is in agreement with many previous studies that reported an increased frequency of Entamoeba gingivalis colonization among people with bad oral hygiene. Improper oral care encourages inflammation of the mucous membrane, gingival diseases, and caries. In addition, it favors the accumulation of food residue and the development of dental plaque, which constitutes an excellent base for the growth of this protozoan. This explains the significantly higher rate of Entamoeba gingivalis in cases presenting with gingivitis in the current work. ${ }^{28-33}$

Concerning diabetes mellitus and smoking as other risk factors for periodontitis, Ibrahim and Abbas, and Nocito et al. found that there was a higher rate of Entamoeba gingivalis in diabetic patients. ${ }^{4,34}$ On the contrary, in our study, there was no significant difference in the occurrence of Entamoeba gingivalis between diabetic and non-diabetic patients, which may be due to our relatively smaller sample size. Regarding the relationship between smoking and Entamoeba gingivalis, no significant difference was observed between smokers and nonsmokers. This result is consistent with Albuquerque et al. who revealed no correlation between smoking, and the incidence of this protozoa. 17

Regarding the demographic data, there are controversies concerning the distribution of infection in relation to sex. In the present study, the percentage of occurrence in females was higher than in males $167.7 \%$ vs $32.3 \%$ respectively). While Gharavi et al. showed that both sexes were equally infected with this parasite, Al- Najar and Adnan showed that the percentage of occurrence in males was higher than in females $(38.4 \%$ and $28.5 \%$ respectively). ${ }^{26,21}$ In accordance, studies by Ullah et al. and Maybodi et al. showed a higher prevalence in males. ${ }^{35,14}$ Furthermore, comparing the age of our subjects, the rate of Entamoeba gingivalis occurrence ranged between the age

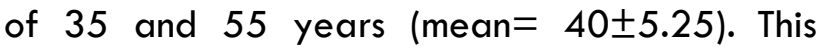
result is consistent with that recorded by Wantland and Laver and Al- Najar and Adnan who found an increased rate of occurrence among patients of up to 40 years of age.36,21 Furthermore, Gharavi et al. has noticed that amoebae colonization is related to an age higher than 20 years. ${ }^{26}$ Contrarily, Albuquerque et al. and Maybodi et al. stated that no relationship was noticed between the age and colonization of Entamoeba gingivalis. ${ }^{17,14}$

Regarding the level of education, our results showed an insignificant difference between well-educated and low educated subjects for Entamoeba gingivalis colonization. In contrast to our results, Hamad et al. found a positive relationship between the presence of the parasite in the mouth and illiteracy or low education level. ${ }^{37}$ Moreover, in the present study, no statistically significant difference between working and non-working patients was observed.

Interestingly, one in vitro study reported that Aggregatibacter actinomycetemcomitans ( $A$. actinomycetemcomitans) was affected by the presence of unidentified oral amoebae. The amoebae enhanced the growth of $A$. actinomycetemcomitans in media which otherwise failed to meet nutritional requirements. ${ }^{38}$ Indeed, A. actinomycetemcomitans is related to a group of bacteria that are associated with biofilm in gingivitis and chronic periodontitis. ${ }^{39}$ Further studies are recommended to clarify the potential interaction between this perio-pathogenic bacterium and Entamoeba gingivalis which is a very common parasite among humans. This hypothesis may clarify the perio-pathogenic role of Entamoeba gingivalis. Amoebae, in particular, use bacteria as a food source but some bacteria may survive phagocytosis and multiply within the amoebae. This may be an explanation for refractory cases as the bacteria harbored inside the amoebae could be protected from the immune system defense mechanisms or antibiotics which may be prescribed as a part of therapy during treatment of periodontitis. In the absence of periodontal disease treatments which might eliminate Entamoeba gingivalis, bacteria sheltered within the amoebae could exit and recolonize the tissues and possibly create a 
refractory case. Thus, anti-parasitic therapy in humans is another suggested treatment modality for periodontal diseases. However, more investigations are required in order to reach sound conclusions regarding the etiological link between Entamoeba gingivalis and periodontal disease. 38,40

To conclude, although the exact contribution of Entamoeba gingivalis to periodontal diseases is not absolutely obvious, our study suggests a perio-pathogenic role of Entamoeba gingivalis in relation to gingivitis. This highlights the potential for an associated pathology and accordingly may warrant a new modality for controlling the disease. Intervention studies on animal models using anti-parasitic treatment and follow up may provide further evidence regarding the etiologic link between Entamoeba gingivalis and periodontal diseases. Further studies are required to investigate the variable risk factors of periodontal diseases in relation to the occurrence of Entamoeba gingivalis.

In light of our investigation, Entamoeba gingivalis may have a role in the pathogenesis of periodontal diseases. Further experimentation is needed to better clarify the etiologic link between the parasite and periodontal diseases, which in turn might be helpful in the treatment of this prevalent worldwide oral disease.

\section{References}

1. Yucel-Lindberg L, Båge T. (2013). Inflammatory mediators in the pathogenesis of periodontitis. Expert Rev Mol Med. 2013; 15:e7. https://doi.org/10.1017/erm.2013.8

2. Bonner $M$, Fresno $M$, Gironès $N$, Guillén $N$, Santi-Rocca J. Reassessing the Role of Entamoeba gingivalis in Periodontitis. Front Cell Infect Microbiol. 2018; 8:379. https://doi.org/10.3389/fcimb.2018.00 003

3. Bhaijee F, Bell D. Entamoeba gingivalis in Acute Osteomyelitis of the Mandible. Case Rep Med. 2011; 2011:357301. https://doi.org/10.1155/2011/35730 3

4. Ibrahim S, Abbas R. Evaluation of Entamoeba gingivalis and Trichomonas tenax in patients with periodontitis and gingivitis and its correlation with some risk factors. Journal of Baghdad College of Dentistry. 2012; 24(3):158-162.

5. García G, Ramos F, Martínez-Hernández F, Hernández L, Yáñez J., Gaytán P. A new subtype of Entamoeba gingivalis: " $E$. gingivalis ST2, kamaktli variant." Parasitol Res. 2018; $117(4): 1277-1284$. https://doi.org/10.1007/s00436-0185798-6

6. Abramson $\mathrm{JH}$. The cornell medical index as an epidemiological tool. Am J Public Health Nations Health. 1966; 56(2):287298.

7. Silness J, Loe H. Periodontal disease in pregnancy. II. Correlation between oral hygiene and periodontal condition. Acta Odontol Scand. 1964; 22:121-35.

8. Löe H. The Gingival Index, the Plaque Index and the Retention Index Systems. $J$ Periodontol. 1967; 38(6):Suppl:610-16. https://doi.org/10.1902/jop.1967.38.6. 610

9. Murakami S, Mealey BL, Mariotti A, Chapple ILC. Dental plaque-induced gingival conditions. J Periodontol. 2018; 89 Suppl 1:S17-S27. https://doi.org/10.1002/JPER.17-0095

10. Tonetti MS, Greenwell H, Kornman KS. Staging and grading of periodontitis: Framework and proposal of a new classification and case definition. $J$ Periodontol. 2018; 89 Suppl 1: S159S172.

https://doi.org/10.1002/JPER.18-0006

11. El-Naggar SM, El-Bahy MM, Abd Elaziz J, El-Dardiry MA. Detection of protozoal parasites in the stools of diarrhoeic patients using different techniques. J Egypt Soc Parasitol. 2006; 36(1):7-22.

12. Kim H, Yoo SB, Choe JY, et al. Detection of ALK gene rearrangement in nonsmall cell lung cancer: a comparison of fluorescence in situ hybridization and chromogenic in situ hybridization with correlation of ALK protein expression. $J$ Thorac Oncol. 2011; 6(8):1359-66. https://doi.org/10.1097/JTO.0b013e3 $3182 \mathrm{cfc} 73$

13. Bailey VL, Bilskis CL, Fansler SJ, McCue LA, Smith JL, Konopka A. Measurements of microbial community activities in 
individual soil macroaggregates. Soil Biology and Biochemistry. 2012; 48:192195.

https://doi.org/10.1016/i.soilbio.2012. 01.004

14. Maybodi FR, Ardakani AH, Bafghi AF, Ardakani AH, Zafarbakhsh A. The Effect of Nonsurgical Periodontal Therapy on TrichomonasTenax and Entamoeba

Gingivalis in Patients with Chronic Periodontitis. J Dent (Shiraz). 2016; $17(3): 171-176$.

15. Burt B, Research, Science and Therapy Committee of the American Academy of Periodontology. Position paper: epidemiology of periodontal diseases. J Periodontol. 2005; 76(8):1406-19. https://doi.org/10.1902/jop.2005.76.8. 1406

16. Bonner $M$, editor. Medical Implication of Oral Amoebiasis. 5th International Congress on Tropical Medicine and International Health. Amsterdam; Canada. Amsterdam: The Netherlands; 2007. pp. 24-28. Available at: www.calameo.com/books/ $0000884407729 \mathrm{fb} 05 \mathrm{e} 363$

17. Albuquerque RLC, Melo CM, Santana WA, Ribeiro JL, Silva FA. Incidence of Entamoeba gingivalis and Trichomonas tenax in samples of dental biofilm and saliva from patients with periodontal disease. Rev Gaúcha Odontol. 2011; 59(1):35-40.

18. Bonner M, Amard V, Bar-Pinatel $C$, et al. Detection of the amoeba Entamoeba gingivalis in periodontal pockets. Parasite. 2014; 21(30):1-9. https://doi.org/10.1051/parasite/201 2014

19. Gardner BB, Del Junco DJ, Fenn J, Hengesbaugh JH. Comparison of direct wet mount and trichrome staining techniques for detecting Entamoeba species Trophozoites in stools. J Clin Microbiol. 1980; 12(5):656-658.

20. Goldsmid JM, Gericke J. Entamoeba gingivalis in Tasmania. Trans $R$ Soc Trop Med Hyg. 1982; 76(5):703.

21. Al- Najar S, Adnan E. The first record of Entamoeba gingivalis in Iraqi patients. J Fac Med Baghdad. 1986; 28(2):73-80.
22. Glebski J, Nierychlewska A, Reczyk J. Extensiveness of occurrence of protozoa in man's oral cavity. Wiad Parazytol. 1975; $21(1): 11-14$.

23. Dao AH, Robinson DP, Wong SW. Frequency of Entamoeba gingivalis in human gingival scrapings. Am J Clin Pathol. 1983; 80(3):380-383. https://doi.org/10.1093/ajcp/80.3.380

24. Trim RD, Skinner MA, Farone $M B$, Dubois JD, Newsome AL. Use of PCR to detect Entamoeba gingivalis in diseased gingival pockets and demonstrate its absence in healthy gingival sites. Parasitol Res. 2011; 109(3):857-864. https://doi.org/10.1007/s00436-01 1 2312-9

25. Gharavi MJ, Hekmat S, Ebrahimi A, Jahani MR. Buccal Cavity Protozoa in Patients Referred to the Faculty of Dentisry in Tehran, Iran. Iranian J Parasitol. 2006; 1(1):43-46.

26. Onyido AE, Amadi ES, Olofin I, Onwumma AA, Okoh IC, Chikwendu $\mathrm{Cl}$. Prevalence of Entamoeba gingivalis and Trichomonas tenax among dental patients attending Federal School of Dental Technology and Therapy Clinic, Enugu, Nigeria. Nature and Science. 2011 ; 9(9): 59-62.

27. Al-Saeed WM. Pathogenic effect of Entamoeba gingivalis on gingival tissue of rats. Al-Rafidain Dent J. 2003; 3:70-73.

28. Linke HA, Gannon JT, Obin JN. Clinical survey of Entamoeba gingivalis by multiple sampling in patients with advanced periodontal disease. Int J Parasitol. 1989; 19(7):803-808.

29. Vráblic J, Tomová S, and Catár G. Occurrence of the protozoa, Entamoeba gingivalis and Trichomonas tenax in the mouths of children and adolescents with hyperplastic gingivitis caused by phenytoin. Bratisl Lek Listy. 1992; 93(3):136-140.

30. Cielecka D, Chomicz L, Piekarczyk J, et al. Oral cavity condition and the occurrence of parasitic protozoans in patients with genetic diseases. Acta Parasitologica. 2000; 45(2):107-1 12. 
31. Liu GY, Chen JF, Wen WR, Chen WL, Lin $L Q$, Hong $H$. Experimental study on the pathogenesis of Entamoeba gingivalis. Zhongguo Ji Sheng Chong Xue Yu Ji Sheng Chong Bing Za Zhi. 2001; 19(4):229-232.

32. Derda M, Hadaś $E$, and Antczak E, Woit WJ. Incidence of Entamoeba gingivalis in the oral cavity of students. J Stoma. 2011 ; 64(10):784-795.

33. Norberg CMBM, Ribeiro PC, SatyroCarvalho N, Sanches FG, Carvalho RW, Norberg AN. Entamoeba gingivalis (Gros, 1849) and Trichomonas tenax (Muller, 1773) oral infections in patients from Baixada Fluminense, province of Rio de Janeiro, Brazil. Science Journal of Public Health. 2014; 2(4):288-292. https://doi.org/10.1 1648/i.sjph.20140 201.17

34. Nocito-Mendoza I, Vasconi-Correas MD, Ponce de León-Horianski P, ZderoPandzich M. Entamoeba gingivalis and Trichomonas tenax in diabetic patients. RCOE. 2003; 8(1):19-23.

35. Ullah Z, Khan M, Jan AH, Ali I. Mouth Protozoa in North West Frontier Province of Pakistan - A Study. Pakistan Oral \& Dental Journal. 27(2):245-248.

36. Wantland WW, Laver D. Correlation of Some Oral Hygiene Variables with Age, Sex, and Incidence of Oral Protozoa. J. Dent. Res. 1970; 49(2):293-297. https://doi.org/10.1177/0022034570 0022034570

37. Hamad SS, Mohammad SH, Kader MA. Relationship between the Dental health and prevalence's Trichomonas tenax and
Entamoeba gingivalis among patients attending Dental Clinics in Kirkuk. Journal of University of Babylon. 2012; 20(5):1441-1447.

38. Derderian GM. An in vitro study of the co-cultivation of Actinobacillus actinomycetemocomitans (MS thesis). Indiana University School of Dentistry, Indianapolis; 1991.

39. Socransky SS, Haffajee AD. Periodontal microbial ecology. Periodontol 2000. 2005; 38:135-187. https://doi.org/10.1111/j.16000757.2005.00107.x

40. Greub G, Raoult D. Microorganisms Resistant to Free-Living Amoebae. Clin. Microbiol. Rev. 2004; 17(2):413-433. https://doi.org/10.1128/CMR.17.2.413 $-433.2004$

Conflicts of interest: The authors declared no conflicts of interest related to this work.

\section{Corresponding author:}

Dr. Gihane Gharib Madkour

Associate Professor, Department of Oral

Medicine and Periodontology

Faculty of Dentistry

Cairo University

11 Al Saraya Street, Al Manial

Cairo, Egypt

E-mail: gihane.madkour@dentistry.cu.edu.eg

Phone: +201002555770

Edited by Professor Ahmed Y. Gamal

This is an open access article distributed under the Creative Commons Attribution-

Noncommercial-NoDerivatives 4.0 International (CC BY-NC-ND 4.0) License. 